CERN-TH/2000-157

hep-th/0006019

\title{
A Note on $(\mathrm{D}(p-2), \mathrm{D} p)$ Bound State and Noncommutative Yang-Mills Theory
}

\author{
Donam Youm ${ }^{1}$ \\ Theory Division, CERN, CH-1211, Geneva 23, Switzerland
}

\begin{abstract}
We give a microscopic explanation for the recently observed equivalence among thermodynamics of supergravity solutions for D $p$-branes with or without NS $B$-field and for $\mathrm{D}(p-2)$-branes with vanishing $B$-field and two delocalized transverse directions by showing that these $\mathrm{D}$-brane configurations are related to one another through $T$-duality transformations. This result also gives an evidence for the equivalence among the noncommutative and the ordinary Yang-Mills theories corresponding to the decoupling limits of the worldvolume theories of such D-brane configurations.
\end{abstract}

CERN-TH/2000-157

June, 2000

\footnotetext{
${ }^{1}$ E-mail: Donam.Youm@cern.ch
} 


\section{Introduction}

Recently, much attention has been given to noncommutative Yang-Mills theory (NCYM), after it is realized that NCYM arises naturally in a specific compactification of the matrix theory [1] and in string theories as decoupling limits of the worldvolume theories on D-branes in nonzero $B$-field background [2,3]. It is argued [3] that NCYM and the ordinary Yang-Mills theory (CYM) arise from the same field theory regularized in different ways. The gauge fields of NCYM and CYM are related [3] by requiring the equivalence of the gauge transformations of the two theories. The evidence for the equivalence between the large $N$ limit NCYM and CYM was also given in Refs. [4, 5].

In the spirit of the holographic principle $[6,7,8]$, the bulk supergravity dual description of NCYM was studied in Refs. $[9,10,11]$. In accordance with the conjectured equivalence between NCYM and CYM, it is found out that thermodynamics of near-extremal $\mathrm{D} p$-branes with nonzero $B$-field coincides exactly with that of the corresponding D $p$-brane without $B$-field $[10,11,12,13,14]$. By observing that the near horizon geometry and thermodynamics of the supergravity solution for the $\mathrm{D} p$ brane with nonzero $B$-field are identical to those of the supergravity solution for the $\mathrm{D}(p-2)$-brane with vanishing $B$-field and two of its transverse coordinates delocalized, it is further argued $[15,16]$ that NCYM in $p+1$ dimensions and CYM in $p-1$ dimensions are equivalent.

It is the purpose of this paper to give a microscopic explanation for the equivalence of thermodynamics of various supergravity solutions for the D-brane systems mentioned in the above. We relate such supergravity solutions to microscopic D-brane systems through the boundary state formalism and show that such D-brane systems are related to one another through $T$-duality transformations, implying that the number of microscopic degrees of freedoms of the above mentioned different D-brane configurations are mapped one-to-one to one another under the $T$-duality transformations. We begin by reviewing the relevant ideas and elaborating on their connection in section 2 , for the purpose of preparing for the discussion of the main result of this paper. In section 3 , we show that various D-brane systems whose thermodynamics are shown to coincide with one another are related under the $T$-duality transformations.

\section{General Properties}

The bosonic action for an open string ending on a $\mathrm{D} p$-brane in the constant NS $B$-field background can be written in the following form:

$$
S=\frac{1}{4 \pi \alpha^{\prime}} \int d^{2} \xi\left[\sqrt{-h} h^{\alpha \beta} g_{\mu \nu} \partial_{\alpha} X^{\mu} \partial_{\beta} X^{\nu}-\epsilon^{\alpha \beta} \mathcal{F}_{i j} \partial_{\alpha} X^{i} \partial_{\beta} X^{j}\right]
$$


by going to the gauge in which the NS $B$-field takes the form $B=B_{i j} d X^{i} \wedge d X^{j}$ $(i, j=0, \ldots, p)[17]$. Here, $\mathcal{F}_{i j} \equiv B_{i j}+\partial_{[i} A_{j]}, A_{i}$ is the $U(1)$ gauge field living on the $\mathrm{D} p$-brane worldvolume and $\xi^{\alpha}=(\tau, \sigma)$ is the string worldsheet coordinates. By applying the gauge transformation $B_{i j} \rightarrow B_{i j}+\partial_{i} \Lambda_{j}-\partial_{j} \Lambda_{i}$ and $A_{i} \rightarrow A_{i}-\Lambda_{i}$, under which the action (1) is invariant, one can set $A_{i}$ equal to zero, which we assume from now on. In the $h_{\alpha \beta}=\eta_{\alpha \beta}$ gauge with constant $g_{\mu \nu}$ and $B_{i j}$, the variation of the bosonic open string action (1) with respect to the string coordinates $X^{\mu}$ yields the the following boundary conditions at the ends $\sigma=0, \pi$ of the open string:

$$
\begin{aligned}
g_{i j} \partial_{\sigma} X^{j}+B_{i j} \partial_{\tau} X^{j} & =0, & i, j=0,1, \ldots, p \\
\delta X^{a} & =0, & a=p+1, \ldots, 9
\end{aligned}
$$

The string propagator with this boundary conditions takes the following form $[18,19,3]$ :

$$
\left\langle X^{i}(z) X^{j}\left(z^{\prime}\right)\right\rangle=-\alpha^{\prime}\left[g^{i j} \ln \frac{\left|z-z^{\prime}\right|}{\left|z-\bar{z}^{\prime}\right|}+G^{i j} \ln \left|z-\bar{z}^{\prime}\right|^{2}+\frac{1}{2 \pi \alpha^{\prime}} \theta^{i j} \ln \frac{z-\bar{z}^{\prime}}{\bar{z}-z^{\prime}}\right],
$$

where $z=\tau+i \sigma$ and

$$
\begin{aligned}
G^{i j} & =\left(\frac{1}{g+B}\right)_{S}^{i j}=\left(\frac{1}{g+B} g \frac{1}{g-B}\right)^{i j}, \\
\theta^{i j} & =2 \pi \alpha^{\prime}\left(\frac{1}{g+B}\right)_{A}^{i j}=-2 \pi \alpha^{\prime}\left(\frac{1}{g+B} B \frac{1}{g-B}\right)^{i j},
\end{aligned}
$$

where the subscripts $S$ and $A$ respectively denote the symmetric and the antisymmetric parts of the matrix. $G^{i j}$ is interpreted as the effective metric seen by the open strings and $\theta^{i j}$ has the interpretation as the noncommutativity parameter as can be seen from the following time ordered commutation relation that follows from Eq. (3):

$$
\left[X^{i}(\tau), X^{j}(\tau)\right]=T\left(X^{i}(\tau) X^{j}\left(\tau^{-}\right)-X^{i}(\tau) X^{j}\left(\tau^{+}\right)\right)=i \theta^{i j} .
$$

So, the end-points of the open string target space coordinates associated with nonzero components of the constant $B$ field live in noncommutative space. Namely, in the presence of nonzero $B$-field, the D-brane worldvolume becomes noncommutative. This result was also obtained by the quantization of $X^{i}$ through the analysis of the time-averaged symplectic form on the phase space [20] or through the Dirac bracket quantization procedure [21]. (See also Refs. [22, 23, 24].)

D-branes can be alternatively described by the "boundary states" $[19,25]$ of the "close-string channel" description. The "open-string channel" and the "closed-string channel" descriptions of D-branes are mapped to one another through $\tau \leftrightarrow \sigma$, under which an open string one-loop diagram and a closed string tree diagram are interchanged. The boundary state is given by the product of a matter and a ghost parts, 
each of which is expressed as the product of the bosonic and the fermionic parts. The GSO projection selects specific linear combinations of such boundary states separately for the NS-NS and the R-R sectors. In this paper, we will be mainly concerned with the bosonic matter part $\left|B_{X}\right\rangle$ of the boundary state. By applying the transformation $\tau \leftrightarrow \sigma$ (followed by an appropriate rescaling of $\tau$ and $\sigma$ ) to the boundary conditions (2) on an open string, one obtains the following conditions on $\left|B_{X}\right\rangle$ at $\tau=0$ :

$$
\begin{aligned}
\left.\left(\partial_{\tau} X^{i}+B^{i}{ }_{j} \partial_{\sigma} X^{j}\right)\right|_{\tau=0}\left|B_{X}\right\rangle & =0, & i, j=0,1, \ldots, p \\
\left(\left.X^{a}\right|_{\tau=0}-x^{a}\right)\left|B_{X}\right\rangle & =0, & a=p+1, \ldots, 9 .
\end{aligned}
$$

In the nonzero $B$-field background with some of coordinates compactified on a torus, the oscillator expansion for the closed string target space coordinates is

$$
X^{\mu}=x^{\mu}+w^{\mu} \sigma+\tau g^{\mu \nu}\left(p_{\nu}-B_{\nu \rho} w^{\rho}\right)+\frac{i}{\sqrt{2}} \sum_{n \neq 0}\left[\frac{\alpha_{n}^{\mu}}{n} e^{-i n(\tau-\sigma)}+\frac{\tilde{\alpha}_{n}^{\mu}}{n} e^{-i n(\tau+\sigma)}\right],
$$

where $w^{\mu}$ is zero for noncompact directions and we assume that only the longitudinal components $B_{i j}$ of the two-form potential are nonzero. So, the conditions (6) on $\left|B_{X}\right\rangle$ at $\tau=0$ in terms of the oscillator modes take the following forms:

$$
\begin{aligned}
& \hat{p}^{i}\left|B_{X}\right\rangle=0, \quad\left(\hat{x}^{a}-x^{a}\right)\left|B_{X}\right\rangle=0, \\
& {\left[(\mathbf{1}+B)^{i}{ }_{j} \alpha_{n}^{j}+(\mathbf{1}-B)^{i}{ }_{j} \tilde{\alpha}_{n}^{j}\right]\left|B_{X}\right\rangle=0,} \\
& \left(\alpha_{n}^{a}-\tilde{\alpha}_{-n}^{a}\right)\left|B_{X}\right\rangle=0, \quad \hat{w}^{a}\left|B_{X}\right\rangle=0
\end{aligned}
$$

where $\mathbf{1}$ is the $(p+1) \times(p+1)$ identity matrix.

We now discuss the $T$-duality transformation [26] of closed string theory on $T^{d}$ and its effect on D-brane configurations. The $T^{d}$ part of the canonical Hamiltonian of the closed string is

$$
H=\frac{1}{4 \pi \alpha^{\prime}} \int_{0}^{2 \pi} d \sigma\left(\begin{array}{ll}
X^{\prime} & 2 \pi \alpha^{\prime} P
\end{array}\right) M(E)\left(\begin{array}{c}
X^{\prime} \\
2 \pi \alpha^{\prime} P
\end{array}\right)
$$

where the matrix $M(E)$ determined by $E=g+B$ is given by

$$
M(E)=\left(\begin{array}{cc}
g-B g^{-1} B & B g^{-1} \\
-g^{-1} B & g^{-1}
\end{array}\right)
$$

$X^{\prime}=\partial_{\sigma} X$ and $P=\left(g \partial_{\tau} X+B \partial_{\sigma} X\right) /\left(2 \pi \alpha^{\prime}\right)$ is the conjugate momentum. It appears from Eq. (9) that the Hamiltonian has the $O(d, d, \mathbf{R})$ symmetry, but since the eigenvalues of the operators $\hat{w}^{i}$ and $\hat{p}^{i}$ in the mode expansion (7) take integer values due to the periodicity condition $X^{i} \sim X^{i}+2 \pi$ of the compactified coordinates actually the Hamiltonian is invariant only under the $O(d, d, \mathbf{Z})$ subset $^{2}$.

\footnotetext{
${ }^{2}$ The Hamiltonian for the open string has the same form (9) in terms of the string coordinates and the conjugate momentum and therefore appears to have the same symmetry as the closed string case. However, the $O(d, d, \mathbf{Z})$ target space duality symmetry is not a symmetry of the open string due to the absence of the winding modes.
} 
Under the $O(d, d, \mathbf{Z})$ transformation with the transformation matrix $\left(\begin{array}{ll}a & b \\ c & d\end{array}\right) \in$ $O(d, d, \mathbf{Z})$, the background fields and the oscillator modes transform as

$$
\begin{aligned}
E & \rightarrow E^{\prime}=(a E+b)(c E+d)^{-1} \\
\alpha_{n}(E) & \rightarrow\left(d-c E^{T}\right)^{-1} \alpha_{n}\left(E^{\prime}\right), \quad \tilde{\alpha}_{n}(E) \rightarrow(d+c E)^{-1} \tilde{\alpha}_{n}\left(E^{\prime}\right) .
\end{aligned}
$$

Note, this transformation is valid also for the $n=0$ case, where the oscillator modes are defined as

$$
\alpha_{0}(E) \equiv \frac{1}{\sqrt{2}} G^{-1}(p-E w), \quad \tilde{\alpha}_{0}(E) \equiv \frac{1}{\sqrt{2}} G^{-1}\left(p+E^{T} w\right)
$$

By making use of the equivalence between the closed-string and open-string channel descriptions of D-branes through boundary states, we study the effect of $T$-duality symmetry of closed string theory on D-branes. For this purpose, it is convenient to divide the closed string coordinate mode expansion of the form (7) into the left-moving and the right-moving parts as $X=\frac{1}{2}\left(X_{-}+X_{+}\right)$with

$$
\begin{aligned}
& X_{-}=x+\sqrt{2}(\tau-\sigma) \alpha_{0}+i \sqrt{2} \sum_{n \neq 0} \frac{\alpha_{n}}{n} e^{-i n(\tau-\sigma)}, \\
& X_{+}=x+\sqrt{2}(\tau+\sigma) \tilde{\alpha}_{0}+i \sqrt{2} \sum_{n \neq 0} \frac{\tilde{\alpha}_{n}}{n} e^{-i n(\tau+\sigma)}
\end{aligned}
$$

where the zero modes $\alpha_{0}$ and $\tilde{\alpha}_{0}$ are defined in Eq. (12). The effect of the $O(d, d, \mathbf{Z})$ symmetry transformation of the closed string theory on the open-string channel can be inferred by noting the map $\tau \leftrightarrow \sigma$ (and therefore $\partial_{\tau} \leftrightarrow \partial_{\sigma}$ ) that connects closed and open string channel descriptions of D-branes. The $O(d, d, \mathbf{Z}) T$-duality transformation (11) is generated by the following transformations:

- Factorized dualities $D_{i}$ :

$$
\left(\begin{array}{ll}
a & b \\
c & d
\end{array}\right)=\left(\begin{array}{cc}
I_{d}-e_{i} & e_{i} \\
e_{i} & I_{d}-e_{i}
\end{array}\right)
$$

where $I_{d}$ is the $d \times d$ identity matrix and the $d \times d$ matrix $e_{i}$ has zero entries except for the $(i, i)$-component which is 1 . When the $B$-field is zero and $g$ is diagonal, i.e. $g=\operatorname{diag}\left(R_{1}^{2}, \ldots, R_{d}^{2}\right)$, the factorized duality $D_{i}$ acts as the wellknown large and small radii duality on the $i$-th coordinate, i.e. $R_{i} \rightarrow 1 / R_{i}$ while the remaining radii unchanged. The oscillator modes transform ${ }^{3}$ as $\alpha_{n}^{i} \rightarrow-\frac{1}{R_{i}^{2}} \alpha_{n}^{i}$

\footnotetext{
${ }^{3}$ The extra factor of $1 / R_{i}^{2}$ in the oscillator mode transformation is due to our choice of gauge in which the periodicity of the coordinates $X$ is fixed to be $2 \pi$ and all the information on the size and the shape of the torus is encoded in the background field $E=g+B$. We have chosen such gauge because we wish to consider the general constant background fields $g$ and $B$.
} 
and $\tilde{\alpha}_{n}^{i} \rightarrow \frac{1}{R_{i}^{2}} \tilde{\alpha}_{n}^{i}$, meanwhile the remaining modes $\alpha_{n}^{j}$ and $\tilde{\alpha}_{n}^{j}(j \neq i)$ remain unchanged. This implies the transformation $\partial_{\tau} X^{i} \leftrightarrow \partial_{\sigma} X^{i}$ on the $i$-th closed string coordinate $X^{i}$. From the correspondence between the open string boundary conditions (2) and the conditions (6) on the boundary states, one can see therefore that the Dirichlet and the Neumann boundary conditions of the $i$-th open string coordinate are interchanged. For a general background $E=g+B$, although $E$ transforms in more complicated way, the oscillator modes transform similarly as the diagonal $E$ case, namely $\alpha_{n}^{i} \rightarrow-\frac{1}{g_{i i}} \alpha_{n}^{i}$ and $\tilde{\alpha}_{n}^{i} \rightarrow \frac{1}{g_{i i}} \tilde{\alpha}_{n}^{i}$ with the remaining modes unchanged, and therefore $\partial_{\tau} X^{i} \leftrightarrow \partial_{\sigma} X^{i}$. So, the factorized dualities generally correspond to the usual $T$-duality transformations that transform $\mathrm{D} p$ brane into $\mathrm{D}(p \pm 1)$-brane.

- Basis change of the compactification lattice $\Lambda$, i.e. $E \rightarrow A E A^{T}$ with $A \in$ $G L(d, \mathbf{Z})$ :

$$
\left(\begin{array}{ll}
a & b \\
c & d
\end{array}\right)=\left(\begin{array}{cc}
A & 0 \\
0 & \left(A^{T}\right)^{-1}
\end{array}\right) \text { s.t. } \quad A \in G L(d, \mathbf{Z}) .
$$

Under this transformation, the background fields and the oscillator modes transform as

$$
g \rightarrow A g A^{T}, \quad B \rightarrow A B A^{T}, \quad \alpha_{n}(E) \rightarrow A^{T} \alpha_{n}\left(E^{\prime}\right), \quad \tilde{\alpha}_{n}(E) \rightarrow A^{T} \tilde{\alpha}_{n}\left(E^{\prime}\right) .
$$

So, the derivatives of closed string coordinates transform as

$$
\partial_{\tau} X \rightarrow A^{T} \partial_{\tau} X, \quad \partial_{\sigma} X \rightarrow A^{T} \partial_{\sigma} X
$$

implying that the worldvolume dimensionality of a D-brane does not change under the $G L(d, \mathbf{Z})$ transformation. We see therefore that the D-brane system with the constant $g$ and $B$ and the associated NCYM are equivalent to those with $A g A^{T}$ and $A B A^{T}$, where $A \in G L(d, \mathbf{Z})$.

- Integer " $\Theta$ "-parameter shift of $E$, i.e. $E_{i j} \rightarrow E_{i j}+\Theta_{i j}$ with $\Theta_{i j}=-\Theta_{j i} \in \mathbf{Z}$ :

$$
\left(\begin{array}{ll}
a & b \\
c & d
\end{array}\right)=\left(\begin{array}{cc}
I_{d} & \Theta \\
0 & I_{d}
\end{array}\right) \text { s.t. } \Theta^{T}=-\Theta .
$$

Under this symmetry, the background fields and the oscillator modes transform as

$$
g \rightarrow g, \quad B \rightarrow B+\Theta, \quad \alpha_{n}(E) \rightarrow \alpha_{n}\left(E^{\prime}\right), \quad \tilde{\alpha}_{n}(E) \rightarrow \tilde{\alpha}_{n}\left(E^{\prime}\right) .
$$

implying that closed string coordinates remain unchanged in the form $X=$ $\frac{1}{2}\left(X_{-}+X_{+}\right)$, meanwhile the $B$-field shifts by the integer-valued antisymmetric matrix $\Theta$ as $B \rightarrow B+\Theta$. So, NCYM associated with $\mathrm{D} p$-brane with constant $B$ is equivalent to NCYM associated with $\mathrm{D} p$-brane with $B+\Theta$. In particular, CYM associated with $\mathrm{D} p$-brane without $B$-field is equivalent to NCYM associated with $\mathrm{D} p$-brane in the integer-valued $B$-field background. 


\section{$3 \quad \mathbf{D} p$-Brane with Rank $2 B$ Field and $(\mathbf{D}(p-2), \mathbf{D} p)$ Bound State}

In this section, we restrict our attention to the case of $\mathrm{D} p$-brane with the rank $2 \mathrm{NS}$ $B$-field and $g_{\mu \nu}=\eta_{\mu \nu}$. We choose the non-zero component of the $B$-field to be $B_{p-1, p}$. In this case, the boundary condition (2) for the open string takes the following form:

$$
\begin{aligned}
\partial_{\sigma} X^{i} & =0, & & i=0,1, \ldots, p-2, \\
\partial_{\sigma} X^{i^{\prime}}+B_{i^{\prime} j^{\prime}} \partial_{\tau} X^{j^{\prime}} & =0, & & i^{\prime}, j^{\prime}=p-1, p \\
\delta X^{a} & =0, & & a=p+1, \ldots, 9 .
\end{aligned}
$$

In the corresponding closed-string channel description of such D-brane configuration, the condition on the bosonic matter boundary state at $\tau=0$ is

$$
\begin{aligned}
\left.\partial_{\tau} X^{i}\right|_{\tau=0}\left|B_{X}\right\rangle & =0, & & i=0,1, \ldots, p-2 \\
\left.\left(\partial_{\tau} X^{i^{\prime}}+B_{i^{\prime} j^{\prime}} \partial_{\sigma} X^{j^{\prime}}\right)\right|_{\tau=0}\left|B_{X}\right\rangle & =0, & & i^{\prime}, j^{\prime}=p-1, p \\
\left(\left.X^{a}\right|_{\tau=0}-x^{a}\right)\left|B_{X}\right\rangle & =0, & & a=p+1, \ldots, 9 .
\end{aligned}
$$

The boundary condition of the form (20) can also be achieved by rotating a $\mathrm{D}(p-1)$ brane in the plane defined by one of its longitudinal direction (say, $X^{p-1}$ ) and one of its transverse direction (say, $X^{p}$ ) and then applying the $T$-duality transformation along the $X^{p}$-direction. The rotation angle $\theta$ is then related to the $B$-field as $B_{p-1, p}=\tan \theta$.

To obtain the long distance behavior of the massless bosonic fields in closed string states interacting with the D-brane, we project the boundary state onto the massless bosonic states $[27,28]$. Namely, the long distance fluctuation of a field $\Psi$ in the closed string spectrum is given by $\delta \Psi=\left\langle P^{(\Psi)}|D| B\right\rangle_{\mathrm{NS}, \mathrm{RR}}$, where $P^{(\Psi)}$ is the projector for the

field $\Psi, D=\frac{\alpha^{\prime}}{4 \pi} \int_{|z| \leq 1} \frac{d^{2} z}{|z|^{2}} z^{L_{0}-a} \tilde{z}^{\tilde{L}_{0}-a}(a=1 / 2$ in the NS-NS sector and $a=0$ in the R-R sector) is the closed string propagator and $|B\rangle_{\mathrm{NS}, \mathrm{RR}}$ is the boundary state for the NS-NS or the R-R sector of the closed string. The resulting long distance fluctuation behavior of the massless bosonic fields is

$$
\begin{aligned}
\delta \phi & =\frac{3-p+2 \sin ^{2} \theta}{2 \sqrt{2}} n T_{p} \frac{V_{p+1}}{k_{\perp}^{2}} \\
\delta h_{\mu \nu} & =n T_{p} \frac{V_{p+1}}{k_{\perp}^{2}} \operatorname{diag}(-\mathrm{A}, \mathrm{A}, \ldots, \mathrm{A}, \mathrm{B}, \mathrm{B}, \mathrm{C}, \ldots, \mathrm{C}) \\
\delta B_{p-1, p} & =\frac{\sin 2 \theta}{\sqrt{2}} n T_{p} \frac{V_{p+1}}{k_{\perp}^{2}} \\
\delta A_{01 \ldots p-2}^{(p-1)} & = \pm \sqrt{2} n T_{p} \sin \theta \frac{V_{p+1}}{k_{\perp}^{2}} \\
\delta A_{01 \ldots p}^{(p+1)} & = \pm \sqrt{2} n T_{p} \cos \theta \frac{V_{p+1}}{k_{\perp}^{2}}
\end{aligned}
$$


where $T_{p}=\sqrt{\pi}\left(2 \pi \sqrt{\alpha^{\prime}}\right)^{6-p}$ is the tension of a $\mathrm{D} p$-brane with the unit brane charge, $V_{p+1}$ is the volume of the $\mathrm{D} p$-brane worldvolume, $k_{\perp}^{2}=\sum_{a=p+1}^{9} k_{a}^{2}$ is the square of the transverse momentum and

$$
\mathrm{A}=\left(p-7-2 \sin ^{2} \theta\right) / 8, \quad \mathrm{~B}=\left(p-7+6 \sin ^{2} \theta\right) / 8, \quad \mathrm{C}=\left(p+1-2 \sin ^{2} \theta\right) / 8 .
$$

Here, we multiplied the entire boundary state by an overall factor $n$, which has to be an integer due to the Dirac quantization condition, so that the D-brane can take arbitrary $\mathrm{R}$-R charge. To express the long distance behavior of the massless fields in ordinary space, rather than in momentum space, we apply the following Fourier transformation, valid for $p<D-3$ :

$$
\int d^{p+1} x d^{D-p-1} y \frac{e^{i k_{\perp} \cdot y}}{(D-p-3) r^{D-p-3} \Omega_{D-p-2}}=\frac{V_{p+1}}{k_{\perp}^{2}}
$$

where $r=\sqrt{y^{a} y^{a}}$ is the radial coordinate of the (overall) transverse space and $\Omega_{n}=$ $2 \pi^{(n+1) / 2} / \Gamma((n+1) / 2)$ denotes the area of a unit $n$-sphere $S^{n}$. And then one has to rescale the fields in the following way so that the fields can be canonically normalized:

$$
\varphi=\sqrt{2} \kappa \phi, \quad g_{\mu \nu}=2 \kappa h_{\mu \nu}, \quad \mathcal{B}_{\mu \nu}=\sqrt{2} \kappa e^{\varphi / 2} B_{\mu \nu}, \quad \mathcal{A}=\sqrt{2} \kappa A
$$

where $\kappa$ is the ten-dimensional gravitational constant and $A$ denotes $A^{(p-1)}$ or $A^{(p+1)}$. The resulting long distance behavior of the massless fields exactly reproduces the asymptotic behavior of the following Einstein-frame supergravity solution for the nonthreshold $\mathrm{D} p$ - and $\mathrm{D}(p-2)$-brane bound state constructed in Ref. [29]:

$$
\begin{aligned}
g_{\mu \nu} d x^{\mu} d x^{\nu}= & H^{-\frac{7-p}{8}} h^{-\frac{1}{4}}\left[-d t^{2}+\cdots+d x_{p-2}^{2}+h\left(d x_{p-1}^{2}+d x_{p}^{2}\right)\right] \\
& +H^{\frac{p+1}{8}} h^{-\frac{1}{4}}\left[d y_{1}^{2}+\cdots+d y_{7-p}^{2}\right] \\
e^{2 \varphi}= & H^{\frac{3-p}{2}} h, \quad \mathcal{B}=\left(1-H^{-1}\right) h \cos \theta \sin \theta d x^{p-1} \wedge d x^{p}, \\
\mathcal{A}^{(p-1)}= & \pm\left(1-H^{-1}\right) \sin \theta d t \wedge \cdots \wedge d x^{p-2}, \\
\mathcal{A}^{(p+1)}= & \pm\left(1-H^{-1}\right) h \cos \theta d t \wedge \cdots \wedge d x^{p},
\end{aligned}
$$

where $H=1+\frac{2 \kappa n T_{p}}{(7-p) \Omega_{8-p}} \frac{1}{r^{7-p}}$ and $h^{-1}=\cos ^{2} \theta+H^{-1} \sin ^{2} \theta$. So, we see that the $\mathrm{D} p$ brane with the rank 2 constant $B$-field is described in long distance region by the $\mathrm{D} p$ and $\mathrm{D}(p-2)$-brane bound state.

Just by considering the boundary condition (20) on the open string coordinates, it appears that the $B$-field component $B_{p-1, p}$ or the rotation angle $\theta$ can take an arbitrary value. However, it turns out that $B_{p-1, p}=\tan \theta$ can take only discrete values determined by the Dirac quantization condition for the brane charge (density). From Eq. (22), one can see that the charge densities for the $\mathrm{D}(p-2)$ - and the $\mathrm{D} p$-branes are $q_{p-2}= \pm \sqrt{2} V_{2} n T_{p} \sin \theta$ and $q_{p}= \pm \sqrt{2} n T_{p} \cos \theta$, where $V_{2}$ is the volume of the 
$\left(x_{p-1}, x_{p}\right)$-plane, which is transverse to the $\mathrm{D}(p-2)$-branes but is longitudinal to the $\mathrm{D} p$-branes. Since the charge density of $\mathrm{D} p$-brane takes only values which are integer multiples of the fundamental $\mathrm{D} p$-brane charge density given by $\mu_{p}=\sqrt{2} \sqrt{\pi}\left(2 \pi \sqrt{\alpha^{\prime}}\right)^{3-p}$, we see that $q_{p}=N_{p} \sqrt{2} \sqrt{\pi}\left(2 \pi \sqrt{\alpha^{\prime}}\right)^{3-p}$, where $N_{p} \in \mathbf{Z}$ is the total number of D $p$-branes. We note that the relative transverse directions $x_{p-1}$ and $x_{p}$, which are transverse to the $\mathrm{D}(p-2)$-branes, are delocalized and therefore there are infinitely many fundamental $\mathrm{D}(p-2)$-branes (with the charge density $\left.\mu_{p-2}=\sqrt{2} \sqrt{\pi}\left(2 \pi \sqrt{\alpha^{\prime}}\right)^{1-p}\right)$ packed on the $\left(x_{p-1}, x_{p}\right)$-plane. However, there are finite numbers (say $\left.n_{p-2}\right)$ of $\mathrm{D}(p-2)$-branes per $\left(2 \pi \sqrt{\alpha^{\prime}}\right)^{2}$ area of this 2-plane (Cf. Refs. [30, 31]). So, the total number of $\mathrm{D}(p-2)$ branes is $N_{p-2}=n_{p-2} V_{2} /\left(2 \pi \sqrt{\alpha^{\prime}}\right)^{2}$ and the total charge density is $q_{p-2}=N_{p-2} \mu_{p-2}$. (The total number $N_{p-2}$ of $\mathrm{D}(p-2)$-branes is finite [infinite], if the volume $V_{2}$ of the 2-plane is finite [infinite].) Making use of these facts, we see that the allowed values of the $B$-field are restricted by the Dirac quantization condition as

$$
B_{p-1, p}=\tan \theta=\frac{q_{p-2}}{q_{p}} \frac{1}{V_{2}}=\frac{n_{p-2}}{N_{p}} \quad\left(n_{p-2}, N_{p} \in \mathbf{Z}\right) .
$$

Note, this is valid whether the volume $V_{2}$ is finite or infinite.

Since the (rank 2) $B$-field can take only rational values, one can always set its nonzero components equal to zero by applying the (integer-valued) $T$-duality transformations, as we explain in the following. We consider only the relevant 2-dimensional part of the $\mathrm{D} p$-brane worldvolume associated with non-zero components $B_{p-1, p}=-B_{p, p-1}=$ $n_{p-1} / N_{p}$ of the $B$-field, i.e. we consider the $O(2,2, \mathbf{Z}) T$-duality transformation. The first step is to make the $B$-field take an integer value by applying the $T$-duality transformation with the $O(2,2, \mathbf{Z})$ matrix of the form (15). We can achieve this, for example, by choosing the entries of the $G L(2, \mathbf{Z})$ matrix $A$ in Eq. (15) to be integer multiples of $N_{p}$. The second step is to transform away the resulting integer valued non-zero $B$-field components by applying the integer " $\Theta$ "-parameter shift $T$-duality transformation with the transformation matrix of the form (18). We choose the $2 \times 2$ antisymmetric matrix $\Theta$ in Eq. (18) to be the negative of the $B$-field transformed through the first step. We note that the $T$-duality transformations that we applied in the above steps do not change the worldvolume dimensionality of the D-brane. So, we have related a $\mathrm{D} p$-brane system with constant rank $2 B$-field to a $\mathrm{D} p$-brane system without $B$-field. This result implies the equivalence between the NCYM and CYM associate with such D-brane systems. This result also explains the microscopic origin of the equivalence between the thermodynamics of the supergravity solutions for $\mathrm{D} p$-branes with nonzero $B$-field and those with vanishing $B$-field $[10,11,12,13,14,16]$. Namely, the microscopic degrees of freedom of such brane configurations responsible for the thermodynamics are mapped one-to-one under the $T$-duality transformation mentioned above.

We notice from the above that different $\mathrm{D} p$-brane systems with the non-zero $B$-field component $B_{p-1, p}=\tan \theta=n_{p-2} / N_{p}$ with the fixed number $N_{p}$ of D $p$-branes but with 
the different number densities $n_{p-2}$ of $\mathrm{D}(p-2)$-branes (and therefore different values of $B_{p-1, p}$ or $\theta$ ) can be mapped under the $T$-duality transformations to the same number of $\mathrm{D} p$-branes without the $B$-field. Therefore, the stringy microscopic degrees of freedom for these different D-brane systems are in one-to-one correspondence under the $T$ duality transformations. This gives the microscopic explanation for the $\theta$ independence of the thermodynamic quantities for the nonextreme supergravity solutions for the $(\mathrm{D}(p-2), \mathrm{D} p)$ bound states, which was previously observed in Refs. [10, 11, 12, 13, 14]. In particular, this implies the equivalence of the two extreme limits corresponding to the cases $\theta=0$ and $\theta=\pi / 2$ to the case with a finite nonzero $\theta$. The $\theta=0$ case is just the $\mathrm{D} p$-branes without $B$-field, i.e. $B_{p-1, p}=\tan \theta=0$. In the $\theta=\pi / 2$ case (i.e. $\left.B_{p-1, p}=\tan \theta=\infty\right)$, there are infinitely many $\mathrm{D}(p-2)$-branes per $\left(2 \pi \sqrt{\alpha^{\prime}}\right)^{2}$ area of the $\left(x_{p-2}, x_{p}\right)$-plane, i.e. $n_{p-2}=\infty$. In this case, the second term in the second line of the open string boundary condition in Eq. (20) dominates, thereby the boundary condition (20) becoming that of open strings attached to $\mathrm{D}(p-2)$-branes. From this, one can see the equivalence of the system of $\mathrm{D} p$-branes with nonzero constant rank $2 B$-field and the system of infinitely many $\mathrm{D}(p-2)$-branes densely stacked on the 2-dimensional plane in the transverse space (thereby the $\mathrm{D}(p-2)$-branes becoming delocalized on the 2-plane), which was previously conjectured [32, 33, 34, 35].

We comment on the decoupling limit of the D-brane worldvolume theories. The NCYM decoupling limit is defined as the limit in which $\alpha^{\prime} \sim \varepsilon^{\frac{1}{2}} \rightarrow 0$ and $g_{i j} \sim \varepsilon \rightarrow 0$ such that $G^{i j}$ and $\theta^{i j}$ in Eq. (4), including $B_{\mathrm{SW}}=B /\left(2 \pi \alpha^{\prime}\right)$, are held fixed [3]. (Note, the difference in the convention of the $B$-field $B_{i j}$ in this paper from the one $B_{\mathrm{SW} i j}$ in Ref. [3].) So, in the NCYM decoupling limit, the $B$-field behaves as

$$
B_{p-1, p}=\tan \theta=\frac{n_{p-2}}{N_{p}}=\frac{\tilde{b}}{\alpha^{\prime}},
$$

with $\alpha^{\prime} \rightarrow 0$ and the noncommutative parameter $\tilde{b}$ held fixed. So, in order for the noncommutative effect on the D-brane worldvolume to survive in the decoupling limit, $B_{p-1, p}$ and therefore the number density $n_{p-2}$ of $\mathrm{D}(p-2)$-branes have to go to infinity. The total number of the $\mathrm{D}(p-2)$-branes is given by $N_{p-2}=n_{p-2} V_{2} /\left(2 \pi \sqrt{\alpha^{\prime}}\right)^{2}$. Note, the NCYM decoupling limit condition on the coordinates $x_{p-1, p}$ of the supergravity solution $(26)$ is $x_{p-1, p}=\frac{\alpha^{\prime}}{\tilde{b}} \tilde{x}_{p-1, p}$ such that $\tilde{x}_{p-1, p}$ are held fixed $[9,36,11]$. So, the total number of $\mathrm{D}(p-2)$-branes is reexpressed as

$$
N_{p-2}=\alpha^{\prime} n_{n-2} \tilde{V}_{2} /\left(4 \pi^{2} \tilde{b}^{2}\right),
$$

where we used the relation $\tilde{V}_{2}=\left(\frac{\alpha^{\prime}}{\tilde{b}}\right)^{2} V_{2}$. From Eq. (28), we see that the $\mathrm{D}(p-2)$ brane number density goes to infinity as $n_{n-2} \sim 1 / \alpha^{\prime}$. So, even if the $\mathrm{D}(p-2)$-brane number density $n_{p-2}$ diverges in the NCYM decoupling limit, the total number $N_{p-2}$ of the $\mathrm{D}(p-2)$-branes can be finite, if $\tilde{V}_{2}<\infty$. As pointed out in the above, in 
the $n_{p-2} \rightarrow \infty$ limit the open string boundary condition (20) reduces to that for the open string ending on $\mathrm{D}(p-2)$-brane, meaning that the long distance behavior of the massless bosonic fields in the closed string states interacting with such D-brane system, obtained from the boundary state through the projection, reproduces that of the supergravity solution for the $\mathrm{D}(p-2)$-branes. Therefore, in the NCYM decoupling limit, the system of $\mathrm{D} p$-branes with rank 2 constant $B$-field reduces to the system of $N_{p-2}$ numbers of $\mathrm{D}(p-2)$-branes, which are densely packed on the 2-plane associated with non-zero components of the $B$-field, implying the equivalence of NCYM in $p+1$ dimensions and CYM with the gauge group $U\left(N_{p-2}\right)$ in $p-1$ dimensions [16]. The rank $N_{p-2}$ of the gauge group is determined by $N_{p}, \tilde{b}$ and $\tilde{V}_{2}$ through Eqs. (28) and (29). When $\tilde{V}_{2}=\infty$, the total number $N_{p-2}$ of the $\mathrm{D}(p-2)$-branes is infinite and therefore NCYM in $p+1$ dimensions is equivalent to CYM with the gauge group $U(\infty)$ in $p-1$ dimensions [15], as pointed out in Ref. [16]. The equivalence of the various D-brane configurations connected through the $T$-duality transformations, on which we elaborated in the previous paragraphs, therefore gives a microscopic explanation for the equivalence among the following gauge theories: (i) CYM in $p+1$ dimensions associated with the decoupling limit of $\mathrm{D} p$-branes without $B$-field, $(i i)$ NCYM in $p+1$ dimensions associated with the NCYM decoupling limit of $\mathrm{D} p$-branes with nonzero constant rank $2 B$-field, (iii) CYM with the gauge group $U\left(N_{p-2}\right)$ or $U(\infty)$ in $p-1$ dimensions.

\section{References}

[1] A. Connes, M.R. Douglas and A. Schwarz, "Noncommutative geometry and matrix theory: Compactification on tori," JHEP 9802 (1998) 003, hep-th/9711162.

[2] M.R. Douglas and C. Hull, "D-branes and the noncommutative torus," JHEP 9802 (1998) 008, hep-th/9711165.

[3] N. Seiberg and E. Witten, "String theory and noncommutative geometry," JHEP 9909 (1999) 032, hep-th/9908142.

[4] D. Bigatti and L. Susskind, "Magnetic fields, branes and noncommutative geometry," hep-th/9908056.

[5] G. Arcioni and M.A. Vazquez-Mozo, "Thermal effects in perturbative noncommutative gauge theories," JHEP 0001 (2000) 028, hep-th/9912140.

[6] G. 't Hooft, "Dimensional reduction in quantum gravity," gr-qc/9310026.

[7] L. Susskind, "Strings, black holes and Lorentz contraction," Phys. Rev. D49 (1994) 6606, hep-th/9308139.

[8] L. Susskind, "The World as a hologram," J. Math. Phys. 36 (1995) 6377, hepth/9409089. 
[9] A. Hashimoto and N. Itzhaki, "Non-commutative Yang-Mills and the AdS/CFT correspondence," Phys. Lett. B465 (1999) 142, hep-th/9907166.

[10] J.M. Maldacena and J.G. Russo, "Large $N$ limit of non-commutative gauge theories," JHEP 9909 (1999) 025, hep-th/9908134.

[11] M. Alishahiha, Y. Oz and M.M. Sheikh-Jabbari, "Supergravity and large $N$ noncommutative field theories," JHEP 9911 (1999) 007, hep-th/9909215.

[12] J.L. Barbon and E. Rabinovici, "On $1 / N$ corrections to the entropy of noncommutative Yang-Mills theories," JHEP 9912 (1999) 017, hep-th/9910019.

[13] R. Cai and N. Ohta, "On the thermodynamics of large $N$ non-commutative super YangMills theory," Phys. Rev. D61 (2000) 124012, hep-th/9910092.

[14] T. Harmark and N.A. Obers, "Phase structure of non-commutative field theories and spinning brane bound states," JHEP 0003 (2000) 024, hep-th/9911169.

[15] J.X. Lu and S. Roy, " $(p+1)$-dimensional noncommutative Yang-Mills and D(p-2) branes," hep-th/9912165.

[16] R. Cai and N. Ohta, "Noncommutative and ordinary super Yang-Mills on ( $\mathrm{D}(p-2), \mathrm{D} p)$ bound states," JHEP 0003 (2000) 009, hep-th/0001213.

[17] E. Witten, "Bound states of strings and p-branes," Nucl. Phys. B460 (1996) 335, hepth/9510135.

[18] E.S. Fradkin and A.A. Tseytlin, "Nonlinear electrodynamics from quantized strings," Phys. Lett. B163 (1985) 123.

[19] C.G. Callan, C. Lovelace, C.R. Nappi and S.A. Yost, "String loop corrections to beta functions," Nucl. Phys. B288 (1987) 525; "Adding holes and crosscaps to the superstring," Nucl. Phys. B293 (1987) 83; "Loop corrections to superstring equations of motion," Nucl. Phys. B308 (1988) 221; A. Abouelsaood, C.G. Callan, C.R. Nappi and S.A. Yost, "Open strings in background gauge fields," Nucl. Phys. B280 (1987) 599.

[20] C. Chu and P. Ho, "Noncommutative open string and D-brane," Nucl. Phys. B550 (1999) 151, hep-th/9812219.

[21] F. Ardalan, H. Arfaei and M.M. Sheikh-Jabbari, "Dirac quantization of open strings and noncommutativity in branes," hep-th/9906161.

[22] M.M. Sheikh-Jabbari, "More on mixed boundary conditions and D-branes bound states," Phys. Lett. B425 (1998) 48, hep-th/9712199.

[23] F. Ardalan, H. Arfaei and M.M. Sheikh-Jabbari, "Mixed branes and M(atrix) theory on noncommutative torus," hep-th/9803067.

[24] F. Ardalan, H. Arfaei and M.M. Sheikh-Jabbari, "Noncommutative geometry from strings and branes," JHEP 9902 (1999) 016, hep-th/9810072.

[25] J. Polchinski and Y. Cai, "Consistency of open superstring theories," Nucl. Phys. B296 (1988) 91.

[26] A. Giveon, M. Porrati and E. Rabinovici, "Target space duality in string theory," Phys. Rept. 244 (1994) 77, hep-th/9401139, and the references therein. 
[27] P. Di Vecchia, M. Frau, I. Pesando, S. Sciuto, A. Lerda and R. Russo, "Classical p-branes from boundary state," Nucl. Phys. B507 (1997) 259, hep-th/9707068.

[28] P. Di Vecchia, M. Frau, A. Lerda and A. Liccardo, “( $\mathrm{F}, \mathrm{D} p)$ bound states from the boundary state," Nucl. Phys. B565 (2000) 397, hep-th/9906214.

[29] J.C. Breckenridge, G. Michaud and R.C. Myers, "More D-brane bound states," Phys. Rev. D55 (1997) 6438, hep-th/9611174.

[30] J.X. Lu and S. Roy, "(m,n)-string-like D p-brane bound states," JHEP 9908 (1999) 002, hep-th/9904112.

[31] J. X. Lu and S. Roy, "Non-threshold (F,D p) bound states," Nucl. Phys. B560 (1999) 181, hep-th/9904129.

[32] P.K. Townsend, "D-branes from M-branes," Phys. Lett. B373 (1996) 68, hepth/9512062.

[33] T. Banks, W. Fischler, S.H. Shenker and L. Susskind, "M theory as a matrix model: A conjecture," Phys. Rev. D55 (1997) 5112, hep-th/9610043.

[34] N. Ishibashi, H. Kawai, Y. Kitazawa and A. Tsuchiya, "A large- $N$ reduced model as superstring," Nucl. Phys. B498 (1997) 467, hep-th/9612115.

[35] N. Ishibashi, " $p$-branes from $(p-2)$-branes in the bosonic string theory," Nucl. Phys. B539 (1999) 107, hep-th/9804163.

[36] J.M. Maldacena and J.G. Russo, "Large $N$ limit of non-commutative gauge theories," JHEP 9909 (1999) 025, hep-th/9908134. 